\title{
Motor and somatosensory evoked potentials in hereditary spastic paraplegia
}

Luciana Pelosi, Bernado Lanzillo, Anna Perretti, Lucio Santoro, Lance Blumhardt, Giuseppe Caruso

\begin{abstract}
Motor evoked potentials (MEPs) from the arms and legs to transcranial stimulation of the motor cortex and somatosensory evoked potentials (SSEPs) from stimulation of the nerves of the arms and legs, were recorded in 11 patients with hereditary spastic paraplegia. Electrophysiological abnormalities were found to be distributed differently among the systems examined; the longer the pathway, the higher the incidence and severity of impairment. MEPs from the leg were either absent or clearly reduced or prolonged in all patients. Eight patients showed abnormal cortical SSEPs on stimulation of the leg (absent or reduced responses in four, slowed central conduction velocity in seven), but only two of these patients had abnormal MEPs from the arm (absent responses). Cortical SSEPs on stimulation of the median nerve were reduced in two patients. Mean values of amplitude and central conduction velocity for MEPs and SSEPs from the leg were significantly different between patients and controls. Such differences were not found for either MEPs or SSEPs from the arm. This distribution of abnormalities, which suggests a differential involvement of the spinal pathways, parallels the reported pathological pattern in which degeneration of axons is more common and severe in the motor and sensory fibres supplying the leg.
\end{abstract}

In a group of patients affected by hereditary spastic paraplegia we recorded motor evoked potentials (MEPs) from the arms and legs during transcranial stimulation of the motor cortex and somatosensory evoked potentials (SSEPs) from stimulation of peripheral nerves in the arms and legs. We determined the type and localisation of electrophysiological abnormalities associated with this disorder and compared these abnormalities with the known pathology of the disease. Neurological Science, University of

Liverpool, Liverpool, UK

L Blumhardt

Correspondence to:

Dr Pelosi, Department of Neurological Science, University of Liverpool, Walton Hospital, Rice Lane, L9 1 AE Liverpool, UK

Received 17 October 1990 and in final revised form 5 April 1991.

Accepted 16 April 1991 sive inheritance. In all patients symptoms and signs were characteristic of hereditary spastic paraplegia. Ten patients showed impaired vibration sense in the leg. Seven patients had features consistent with the pure form of hereditary spastic paraplegia. In three siblings with the autosomal dominant form the EEG showed diffuse slowing. Severe distal muscle atrophy was present in one patient with autosomal recessive inheritance.

\section{Methods}

Surface disc electrodes, $11 \mathrm{~mm}$ in diameter (Dantec, 13L29), were used for stimulating and recording. Electrode impedance was below 5000 ohms. Filter bandwidth was $10-2000 \mathrm{~Hz}$, and the analysis time was $100 \mathrm{~ms}$.

In 10 patients transcranial electrical stimulation of the motor cortex was performed with the unifocal low voltage technique. ${ }^{1}$ Seven interconnected electrodes, functioning as the cathode, were applied to the scalp along a line about $2 \mathrm{~cm}$ above the nasion-inion plane. Anodal stimulation of the motor cortex of the leg was performed with a single electrode $1.5 \mathrm{~cm}$ in front of $\mathrm{Cz}$. For the motor cortex of the arm the stimulation site was $6.5 \mathrm{~cm}$ lateral to and $1.5 \mathrm{~cm}$ in front of $\mathrm{Cz}$. Stimulus duration was $0.3 \mathrm{~ms}$ and intensity ranged from 70 to $90 \mathrm{~mA}$. Muscle recordings of the motor evoked potentials (MEPs) were performed from the rectus femoris and tibialis anterior in the leg and the abductor pollicis brevis in the hand during voluntary background contraction. The common peroneal nerve was stimulated at the knee in seven patients and the median nerve at the wrist in 10 patients. Motor action potentials (MAPs) and F-waves were recorded from the anterior tibial and the abductor pollicis brevis muscles, respectively. For both MEPs and MAPs latency was measured to the response onset and amplitude between the most prominent negative and positive peaks. Central conduction time (CCT) from motor cortex to the spinal cord was indirectly calculated on the basis of the F-wave latency. ${ }^{1}$ Central conduction velocity (CCV) from motor cortex to the lower spinal cord segment was calculated by dividing the distance in centimetres between $\mathrm{Cz}$ and the vertebral spine of T12 by the CCT value.

Somatosensory evoked potentials (SSEPs) obtained by independent stimulation of the tibial nerve at the ankle (TN-A) and either tibial (TN-K) or common peroneal nerve $(\mathrm{CPN}-\mathrm{K})$ at the knee were studied in all subjects. Stimulating electrodes were positioned $3 \mathrm{~cm}$ apart along the nerve with the 
cathode proximal. The stimulus (duration $0.2 \mathrm{~ms}$, intensity $2.5-3.0$ times the sensory threshold) produced a moderate but not uncomfortable muscle twitch. Spinal recordings were made from bipolar leads at $\mathrm{L} 3-\mathrm{L} 1$ and T12-T10 and scalp recordings from $\mathrm{Cz}^{\prime}-$ Fpz and $\mathrm{Cz}^{\prime}$-shoulder contralateral to the stimulated side $\left(\mathrm{Cz}^{\prime}=2 \mathrm{~cm}\right.$ behind $\left.\mathrm{Cz}\right)$. The latency of the spinal SSEPs was measured to the onset of the first negative wave and the amplitude from the first negative to the following next positive peak. The latency of the scalp SSEPs was measured to the peak of the first positive potential (P37 for ankle stimulation and P27 for knee stimulation), and the amplitude was measured peak-to-peak from the first positive potential to the next negative wave (P37/N45 for ankle stimulation and P27) N35 for knee stimulation). Peripheral conduction velocity was calculated from the peripheral nerve stimulation point to L3 and central conduction velocity (CCV) from $\mathrm{L} 3$ to $\mathrm{Cz}^{\prime}$ and from $\mathrm{T} 12$ to $\mathrm{Cz}^{\prime}$.

SSEPs from stimulation of the right median nerve at the wrist were studied in all patients. Stimulus characteristics were the same as for the leg. Recordings were performed at Erb's point, the spine of the seventh cervical vertebra (Cv7), and the contralateral hand somatosensory cortex $\left(\mathrm{C}^{\prime}=2 \mathrm{~cm}\right.$ behind and $7 \mathrm{~cm}$ lateral to $\mathrm{Cz}$ ). The reference electrode was placed on the left earlobe for Erb's and C3' recordings and at $\mathrm{Fpz}$ for $\mathrm{Cv} 7$ recording. The latency was measured to the first positive peak of the potential recorded at Erb's point, to N13 peak (Cv7) and to $\mathrm{N} 20$ peak (C3'). Amplitude was measured peak-to-peak from the first positive component to the next negative component of the triphasic potential recorded at Erb's point and from $\mathrm{N} 13$ and $\mathrm{N} 20$ to the respective preceding positive potentials. Peripheral conduction velocity was calculated from the wrist to Erb's point; the N13-N20 interpeak latency was taken to represent central conduction time (CCT).

Normal data were obtained with the same technique as above from $\mathbf{5 7}$ healthy subjects (table 1). Twenty four subjects were examined for motor evoked potentials (14 men and 10 women, mean age 28 , range $20-35$ years) and 33 subjects for somatosensory evoked potentials (21 men and 12 women, mean age 26.4 , range 21-34 years). Values from patients were considered abnormal when they exceeded $2.5 \mathrm{SD}$ limits.

\section{Results}

MEPs were abnormal in all 10 patients on stimulation of the leg motor cortex and in only two patients on stimulation of the arm motor cortex (table 2). These abnormalities consisted mainly of absent or clearly reduced responses (mean amplitude $0.11 \mathrm{mV}, 21 \%$ of mean amplitude in controls) (figures 1 and 2). Slowed $\mathrm{CCV}$ (mean CCV $38.3 \mathrm{~m} / \mathrm{sec}, 63 \%$ of mean value in controls) was found in four patients (figure 2, table 2). On direct stimulation of the common peroneal and median nerves, one patient whose dominant inherited disease was associated with diffuse EEG slowing had a
Table 1 Mean and SD of electrophysiological findings in patients and controls

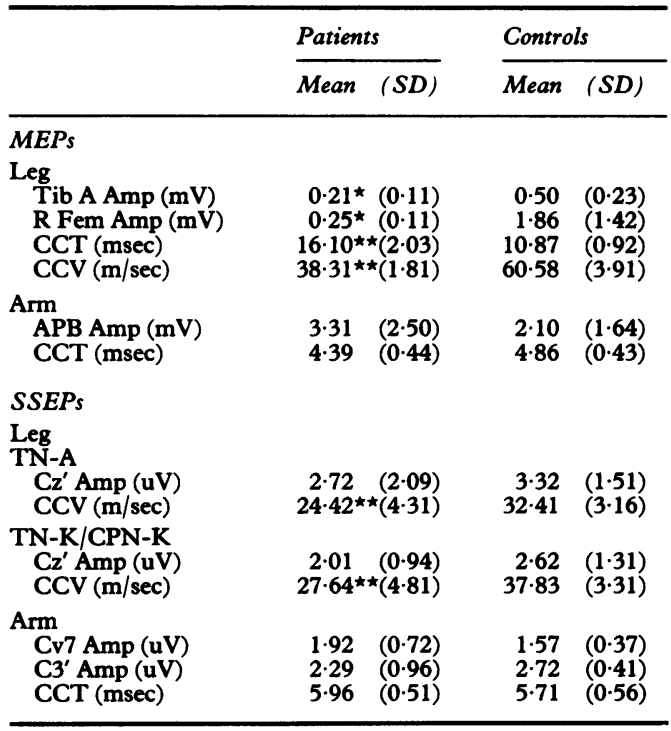

Amp = amplitude; $\quad$ CCT $=$ central conduction time $\mathbf{C C v}=$ central conduction velocity; $\mathbf{A P B}=$ abductor pollicis brevis; Tib $A$ = tibialis anterior; $\mathbf{R}$ Fem = rectus femoris; and $\star \star$ denote significantly different mean values compared with controls $\left(\star_{p}<0.05 ;{ }^{\star \star} p<0.00005\right)$.

moderate increase of distal latencies, while one other patient with severe distal muscle atrophy had low amplitude, moderately delayed MAPs.

SSEPs on stimulation of the leg were abnormal in eight patients (table 2 ). In five, changes were found on stimulation of the TN-A and consisted mainly of absent or poor definition of cortical responses (mean amplitude $0.73 \mathrm{uV}$, $22 \%$ of amplitude in controls) (figure 3 ). Slowed L3-Cz CV (mean CCV $18.7 \mathrm{~m} / \mathrm{sec}$, $58 \%$ of mean CCV in controls) was found in three patients. In two of the five patients and in the three others, slowed $\mathrm{L} 3-\mathrm{Cz}^{\prime} \mathrm{CV}$ was found on either TN or CPN stimulation at the knee (mean CCV $23.3 \mathrm{~m} / \mathrm{sec}, 62 \%$ of mean value in controls) (table 2). Peripheral abnormalities were found in only one of the eight patients and consisted of poorly defined responses over the cauda equina. Abnormalities of the median nerve SSEPs were observed in two patients (amplitude reduction of N20) (table 2).

Mean values of CCV for both MEP and SSEP as well as for MEP amplitude from the leg were significantly lower in patients than in controls (table 1). No significant differences between patients and controls were found for either amplitude or CCV of MEP and SSEP from the arm (table 1).

\section{Discussion}

Histological abnormalities found in patients with pure familial spastic paraplegia consist of axon degeneration mainly involving the longest, large-diameter ascending and descending spinal cord fibre systems. ${ }^{23}$ Degeneration is consistently most severe in the most distal portion of these systems. Spinal roots and peripheral nerves, however, have been found to be normal in both morphological ${ }^{2}$ and electrophysiological studies. ${ }^{4}$ In 18 patients with 
Table 2 Prevalence and type of abnormal electrophysiological findings in patients

\begin{tabular}{|c|c|c|c|c|c|}
\hline & \multirow{2}{*}{\multicolumn{2}{|c|}{ MEPs }} & \multicolumn{3}{|l|}{ SSEPs } \\
\hline & & & \multicolumn{2}{|l|}{ Leg } & \multirow[b]{2}{*}{ Arm } \\
\hline & Leg & Arm & $T N-A$ & $T N-K / C P N-K$ & \\
\hline $\begin{array}{l}\text { No of patients } \\
\text { Absent responses (MEP/cortical SSEP) } \\
\text { Amplitude reduction (MEP/cortical SSEP) } \\
\text { Amplitude reduction (MEP/cortical SSEP) and slowed CCV/ } \\
\text { prolonged CCT }\end{array}$ & $\begin{array}{r}10 \\
6 \\
0 \\
2\end{array}$ & $\begin{array}{r}10 \\
2 \\
0 \\
0\end{array}$ & $\begin{array}{r}11 \\
1 \\
1 \\
2\end{array}$ & $\begin{array}{r}11 \\
0 \\
0 \\
0\end{array}$ & $\begin{array}{r}11 \\
0 \\
2 \\
0\end{array}$ \\
\hline Slowed CCV/prolonged CCT & 2 & 0 & 1 & 5 & 0 \\
\hline No of patients with abnormalities & 10 & 2 & $5^{\star}$ & $5^{\star}$ & 2 \\
\hline
\end{tabular}

‡Two patients in common.
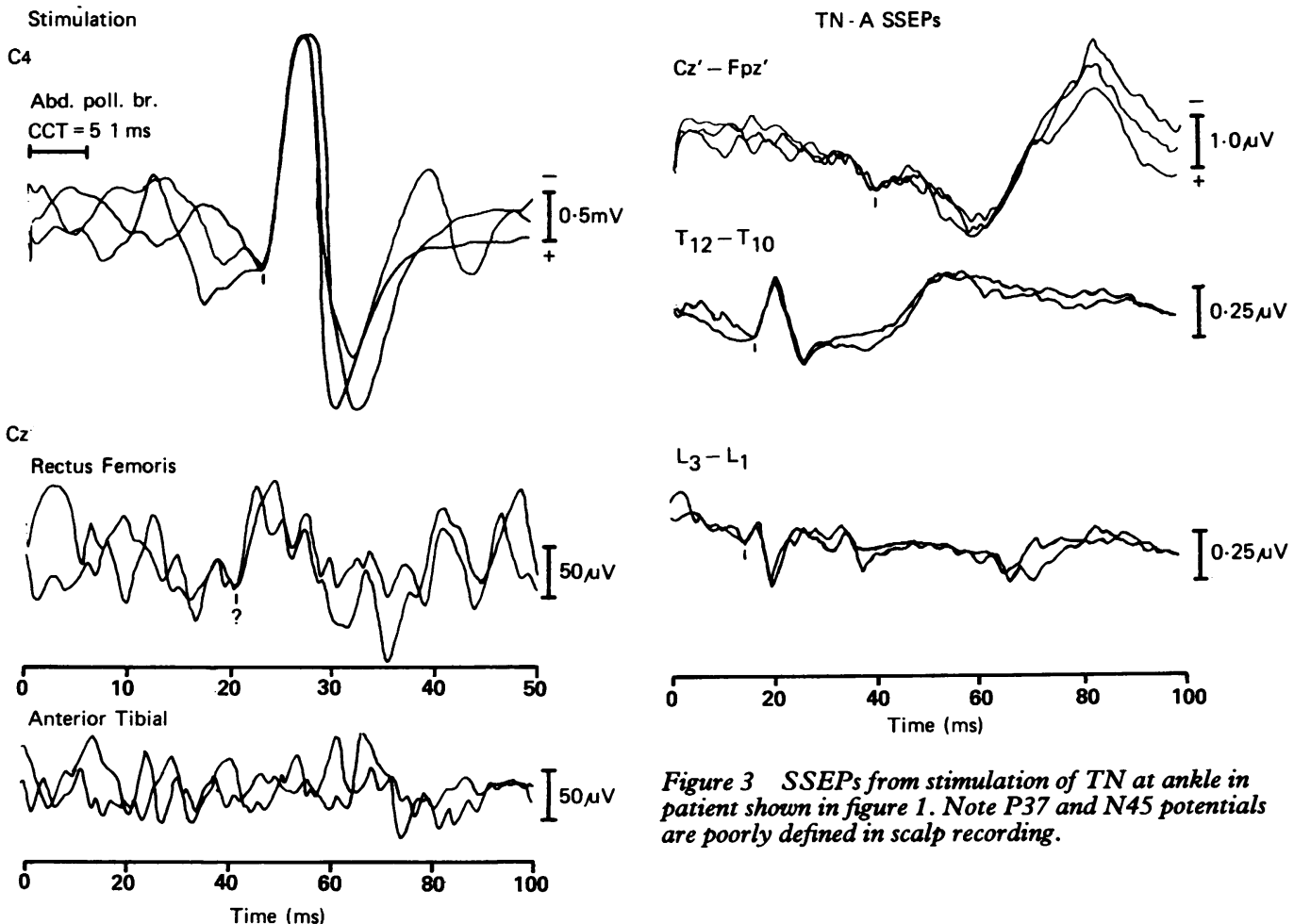

Figure 1 Arm (top trace) and leg (bottom two traces) MEPs from transcranial stimulation of motor cortex in 15 year old patient with autosomal dominant form of hereditary spastic paraplegia. Conduction along central motor pathway (CCT) to arm and amplitude of $M E P s$ from abductor pollicis brevis are normal. MEPs are absent from tibialis anterior and poorly defined from rectus femoris.
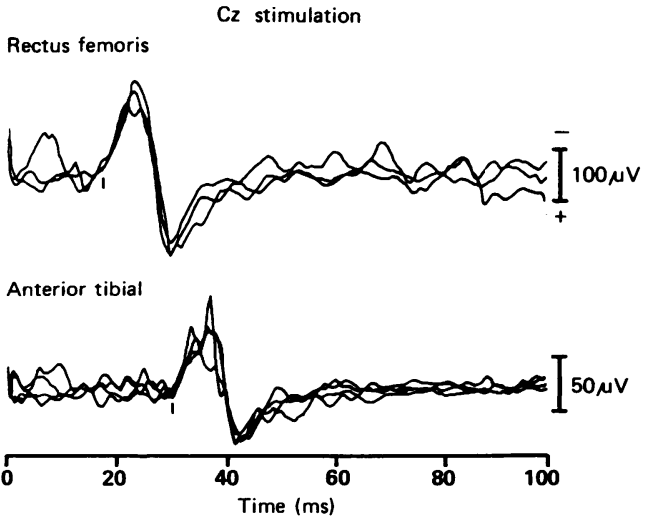

Figure 2 Leg MEPs from transcranial stimulation of motor cortex in 14 year old patient with autosomal dominant form of hereditary spastic paraplegia. Response amplitudes are low and latencies are delayed.

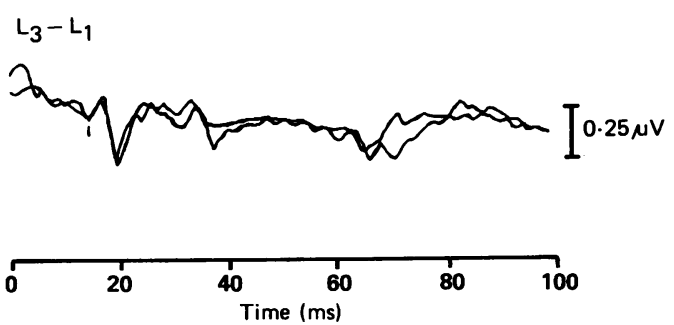

Figure 3 SSEPs from stimulation of $T N$ at ankle in patient shown in figure 1 . Note P37 and N45 potentials are poorly defined in scalp recording.

familial spastic paraplegia, Thomas et al found absent or low amplitude cervical potentials on median nerve stimulation, despite preserved sensory nerve conduction. ${ }^{5}$ This led them to hypothesise a selective distal axonopathy affecting only the central branch of the primary sensory neurons. Subsequent studies of SSEPs, on stimulation of either the arm or leg, appeared to be consistent with this hypothesis. $^{6-8}$ In general, normal findings have been obtained from all arm and leg peripheral nerves (with the exception of three out of 13 tibial nerves examined by Pedersen and Trojaborg ${ }^{6}$ ). In contrast, in each of these studies the cortical SSEPs from leg stimulation in a proportion of patients showed either reduced amplitude ${ }^{8}$ or delayed responses. ${ }^{67}$ SSEPs to median nerve stimulation, however, gave less consistent results. They were normal at all recording levels in 11 out of 13 patients (two with delayed cortical responses) examined by Pederson and Trojaborg ${ }^{6}$ and in three studied by Uncini $e t$ al. ${ }^{8}$ In contrast, 18 patients studied by Thomas et al showed either absent or reduced cervical responses. 5

Transcranial stimulation of the motor cortex in patients with familial spastic paraplegia has 
been performed in two studies: Thompson et al found that MEPs were delayed in one leg of each of the two patients studied"; Claus et al recorded from the arm and found normal responses in eight of 10 of their patients. ${ }^{10}$

Our results suggest a differential involvement in the cord between the fibre systems related to the arm and to the leg. We found that the longer the pathway the higher the incidence and severity of the electrophysiological abnormalities. Thus abnormalities were present along the motor pathways to the leg in all 10 patients and to the arm in only two. In the somatosensory pathways from the leg there were abnormal findings in eight of 11 patients and from the arm in two. These abnormalities correspond to the observed distribution of fibre degeneration, which was always severe in the crossed corticospinal tract at the thoracic level and often observed in the rostral portion of the fasciculus gracilis but was less marked in the pyramidal tract, in the medulla and cervical cord as well as in the fasciculus cuneatus. ${ }^{23}$

The electrophysiological abnormalities in our patients consisted mainly of absent or poorly defined responses (7/11), which are consistent with a disease process primarily due to axonal degeneration. Moderate slowing of $\mathrm{CCV}$ represented half of the observed abnormalities and in one third of the cases was associated with decreased response amplitude. This CCV slowing could be due to both loss of larger faster axons, which would result in conduction along slower fibres, and reduction of afferent impulses to the synapses, which would increase the cell excitation time.

Apart from the patient with severe distal muscle atrophy, in whom the leg MEP abnormalities were predictably associated with changes along the peripheral motor segment (suggesting a concomitant disorder of the spinal motor neurons), all other patients showed that abnormalities were remarkably less common along the peripheral nerves than along the central segments of both motor and sensory pathways. These results are similar to those obtained by others. ${ }^{4-8}$ In our patients, however, both distribution and severity of abnormalities along the central sensory pathways from the leg were dependent on the site of stimulation. In some subjects pathological SSEPs were found on both ankle and knee stimulation; in some cases only on stimulation of the $\mathrm{TN}$ at the ankle; and in others only on stimulation of either the TN or the CPN at the knee. The most severe abnormalities were found on stimulaton of the tibial nerve at the ankle. These results support the hypothesis that stimulation at the ankle or knee results in SSEPs with different relative contributions from the multiple sensory spinal pathways. ${ }^{11}$ They would also be consistent with the finding that axonal degeneration in hereditary spastic paraplegia is often either confined to or more severe in one of the multiple long pathways ascending the spinal cord. This selective involvement of one spinal pathway in patients with hereditary spastic paraplegia could be an additional explanation for the common observation that normal electrophysiological findings along the peripheral nerve coexist with abnormal findings in the central pathways.

1 Rossini PM, Marciani MG, Caramia M, Roma V, Zarola F. Nervous propagation along "central" motor pathways in intact man: characteristics of motor responses to "bifocal" and "unifocal" spine and scalp non-invasive stimulation. Electroenceph Clin Neurophysiol 1985;61:272-86.

2 Schwarz GA, Liu CN. Hereditary familial spastic paraplegia. Further clinical and pathologic observations. Arch Neurol Psychiat (Chic) 1956;75:144-62.

3 Behan WMH, Maia M. Strumpell's familial spastic paraplegia: genetics and neuropathology. $J$ Neurol Neurosurg Psychiatry 1974;37:8-20.

4 McLeod JG, Morgan JA, Reye C. Electrophysiological studies in familial spastic paraplegia. $J$ Neurol Neurosurg Psychiatry 1977;40:611-5.

5 Thomas PK, Jefferys JGR, Smith IS, Loulakakis D. Spinal somatosensory evoked potentials in hereditary spastic somatosensory evoked potentials in hereditary spastic

6 Pedersen L, Trojaborg W. Visual, auditory and somatosensory pathway involvement in hereditary cerebellar ataxia, Friedreich's ataxia and familial spastic paraplegia. Elec troenceph Clin Neurophysiol 1981;52:283-97.

7 Dimitrijevic MR, Lenman JAR, Prevec T, Wheatly K. A study of posterior column function in familial spastic paraplegia. J Neurol Neurosurg Psychiat 1982;45:46-9.

8 Uncini A, Treviso M, Basciani M, Gambi D. Strumpell's familial spastic paraplegia: an electrophysiological demonstration of selective central distal axonopathy. Electro enceph Clin Neurophysiol 1987;66:132-6.

9 Thompson PD, Day BL, Rothwell JC, et al. The interpretation of electromyographic responses to electric stimulation of the motor cortex in diseases of the upper motor neurone. J Neurol Sci 1987;80:91-110.

10 Claus D, Waddy HM, Harding AE, Murray NMF, Thomas PK. Hereditary motor and sensory neuropathies and hereditary spastic paraplegia: a magnetic stimulation study. Ann Neurol 1990;28:43-9.

11 Pelosi L, Cracco JB, Cracco RQ. Conduction characteristics of somatosensory evoked potentials to peroneal, tibial and sural nerve stimulation in man. Electroenceph Clin Neuro physiol 1987;68:287-94. 